médecine/sciences $1994 ; 10: 919$

\title{
La souris dy/dy : une anomalie des membranes basales musculaires ?
}

La mutation $d y$ de la souris est apparue spontanément et se transmet selon le mode autosomique récessif. Elle est responsable, à l'état homozygote, d'un phénotype essentiellement constitué d'une myopathie progressive. Des anomalies de la myélinisation et une stérilité ont également été rapportées. Ces souris meurent entre 2 et 6 mois sans que la cause de la létalité ne soit réellement comprise. Une collaboration scandinave a permis de mettre en évidence chez les souris homozvgotes $d y / d y$ une absence de la chaîne $M$ de la laminine dans les tissus où elle est normalement exprimée, notamment dans le muscle squelettique ainsi que dans le cœur ou les testicules [1]. I.es laminines constituent une famille de protéines des membranes basales, composées généralement de trois chaînes, et représentent les protéines non collagéniques majoritaires de la matrice extracellulaire [2]. La laminine de type $\mathrm{M}$ constitue la principale laminine des membranes basales du muscle strié, du nerf périphérique et du trophoblaste. Cette absence de chaîne $M$ est associée à des fragmentations de la membrane basale musculaire visualisées en microscopie électronique. Dans le muscle de la souris $d y / d y$, parmi les protéines examinées, aucune autre protéine de la matrice extracellulaire ne semble être portée manquante. L'entactine (ou nidogène), qui forme un complexe stable avec la laminine, ainsi que le collagène de type IV, composant majoritaire de ces membranes basales, sont notamment retrouvés en quantité normale dans le muscle de ces souris mutantes. Bien que l'anomalie moléculaire en cause n’ait pas encore été découverte, deux arguments, l'un moléculaire et l'autre issu des études de liaison génétique, font penser que cette absence de laminine $M$ est une conséquence directe de l'altération de son gène. En effet, l'abondance $\mathrm{du}$ transcrit laminine $\mathrm{M}$ est diminuée chez les souris homozvgotes pour la mutation, ce qui pourrait être compatible avec une mutation non-sens. Par ailleurs, les études de liaison génétique avaient montré que le locus morbide était situé sur le chromosome 10 de la souris, très proche du gène I)RP (codant pour la dystrophin related protein encore appelée utrophine). Or, sur la carte génétique humaine, gènes de la DRP et de la laminine sont à proximité l'un de l'autre (respectivement en $6 q 24$ et 6q22-23) ; la comparaison des cartes murine et humaine suggère donc que le gène de la chaîne $M$ de la laminine soit également localisé sur le chromosome 10 de la souris, donc très proche ou équivalent au locus $d y$. I a laminine est indirectement liée à la dvstrophine, par l'intermédiaire d'un complexe de protéines extra-, intra- et sous-membranaires $\left(\mathrm{m} / \mathrm{s} n^{\circ} 10\right.$, vol. 7 , p. 1090). Voici donc, en dehors de la myopathie de Duchenne et de la myopathie récessive autosomique, un troisième syndrome myopathique, chez la souris cette fois, associé à l'absence d'une des protéines de ce complexe. Une diminution de la laminine de type $M$ avait également été décrite antérieurement dans le muscle et le nerf périphérique de patients atteints de myopathie de Fukuvama; cependant, il ne s'agit pas dans cette affection d'une altération directe puisque les études de liaison ont par la suite montré la localisation du gène de la myopathie de Fukuyama sur le chromosome 9 et non sur le $6\left(\mathrm{~m} / \mathrm{s} n^{\circ} 1\right.$, vol. 10 , p. 122). Il reste que l'altération d'un seul des membres de ce complexe musculaire péri-membranaire est à l'origine d'une variété de maladies où le processus myopathique prédomine. Gageons que d'autres protéines du complexe ne resteront pas longtemps orphelines d'affect ion...

H.G.

1. Su H, Christmas P. Wu XR. Wewer L:M, Engrall $\mathrm{E}$. Defective muscle basement membrane and lack of M-laminin in the dvstrophic $d y / d y$ mouse. Proc Natl Acad Sci LSA 1994 ; 91 : 5572-6. 2. Aumaillev M. Verrando P. Structure et pathor logie des membranes basales. médecine/sciences $1993: 9: 926-33$. 